\title{
The Effect of Gusts on Aircraft Wake Vortices ${ }^{1}$
}

\author{
Anton Stephan, Frank Holzäpfel, Stefan Zholtowski \\ Deutsches Zentrum für Luft- und Raumfahrt, Institut für Physik der Atmosphüre, \\ Oberpfaffenhofen, Germany
}

\begin{abstract}
Purpose - Aircraft wake vortices present a potential risk to following aircraft, particularly during final approach and landing, as wake vortices may remain in the flight corridor for a long time. Wind and turbulence are the key factors influencing the wake vortex evolution, but also the wake vortex generation at the aircraft. Flying through a gust influences the wake vortex roll-up process and its evolution. Note that vertical and lateral gusts may affect counterrotating wake vortices differently. Both vortices influence each other by inducing a downward velocity. Disturbances may therefore lead to local vortex tilting and later to a complex three-dimensional deformation. This work employs two different hybrid Reynolds-averaged Navier-Stokes/large-eddy simulation (RANS/LES) approaches to investigate the effect of gusts on wake-vortex evolution. In a one-way coupling a pre-calculated RANS velocity field of the aircraft's near-field is being swept through an LES domain. The effect of a sine gust on the turbulent wake is modelled by manipulating the RANS-field accordingly. As a more sophisticated approach the concept of a two-way coupling is being presented. Here an LES solver is bi-directionally coupled with an unsteady RANS (URANS) solver, exchanging values at every physical time step of the simulation.
\end{abstract}

Design/methodology/approach - A one-way coupling approach of the LES code MGLET and the RANS code TAU is presented to simulate the gust effect on aircraft wake vortices. Additionally the concept of a two-way coupling of these two codes incorporating a coupling module.

Findings - The gust effect of wake vortices subjected to a crosswind can be simulated. The vortex physics is analysed. Unexpected behavior like fast upwind vortex decay is revealed.

Practical implications - The understanding of the aircraft wake vortex physics during landing provides valuable information for wake-vortex advisory systems.

Originality/value - The effect of gust on wake vortices during and after landing has not been studied so far. The hybrid on-way coupling approach is relatively new, as well as the concept of the two way coupling.

Keywords Wake Vortex; hybrid RANS-LES; gust encounter

Paper type Research paper

\section{Introduction}

Wake vortices are generated by lifting surfaces of flying aircraft in a natural way through the creation of lift. The complex wake structures finally roll-up to a vortex system of two counter rotating vortices. The generated two-vortex system can persist for several minutes, possessing a high amount of kinetic energy and thereby posing a potential hazard to following aircraft. In ground proximity transport and decay of the wake vortices are mainly determined by the wind conditions and the interaction with the ground. To avoid wake-vortex encounters, regulatory separation distances between aircraft pairs have to be maintained based on the leading aircraft's weight, which might limit the capacity at the airports. Therefore, the investigation of wake-vortex decay is an important subject in aviation since the 80th, Spalart (1998); Gerz et al. (2002). In recent years, the dynamics of wake vortices after roll-up until decay have been mainly studied by large-eddy simulations (LES) considering the effects of various atmospheric conditions like turbulence, thermal stability and wind shear, Holzäpfel et al. (2001); Misaka et al. (2012). Those studies initialize a vortex pair in the simulation with a constant velocity profile along the flight direction. The interaction with the ground is also simulated for fully rolled-up vortices, Proctor et al. (2000); Georges et al. (2005). In this approach the effects of the touchdown maneuver and the different vortex generation heights above the ground cannot be studied, and thus may not capture the full three-dimensional vortex deformations appearing during ground interaction. Recent developments in Reynolds-averaged Navier-Stokes (RANS)-LES coupling enable an innovative methodology to simulate the aircraft flight through a simulation domain generating a wake in a realistic way, Misaka et al. (2015). For this purpose a high-fidelity steady RANS flow field is swept through the LES domain. A spatial development of the aircraft wake is introduced in the LES.

\footnotetext{
${ }^{1}$ Aircraft Engineering and Aerospace Technology 2017 89:5, 692-702, http://www.emeraldinsight.com/doi/full/10.1108/AEAT-01-2017-0032
} 
Recent technical developments allow the simulation of the entire vortex evolution with a hybrid RANS/LES approach. This includes the roll-up phase as well as the decay phase. The results have revealed valuable insights for en route flight, Misaka et al. (2015), as well as a flight in ground proximity during the landing phase, Stephan et al. (2014). The wake vortex evolution is driven by the complex interaction of many factors like ambient turbulence, turbulent wind, ground interaction, the touchdown maneuver and subsequent end effects. However, as it can be observed when condensation trails form in humid air, marking the vortex cores that adopt helical shapes and quickly disappear after touchdown, the rolled-up vortices are disturbed right from the beginning. They are subjected to strong three dimensional deformations, see Figure 1. Aircraft maneuvers and flying through turbulence and gusts directly influences the aerodynamics of the wing and consequently the wake structure of the aircraft. The aim of this study is

Figure 1 Complex vortex deformations after landing in crosswind situation. Youtube video, Juli, 20th 2016, https://www.youtube.com/watch?v=ViKYFsN3p24

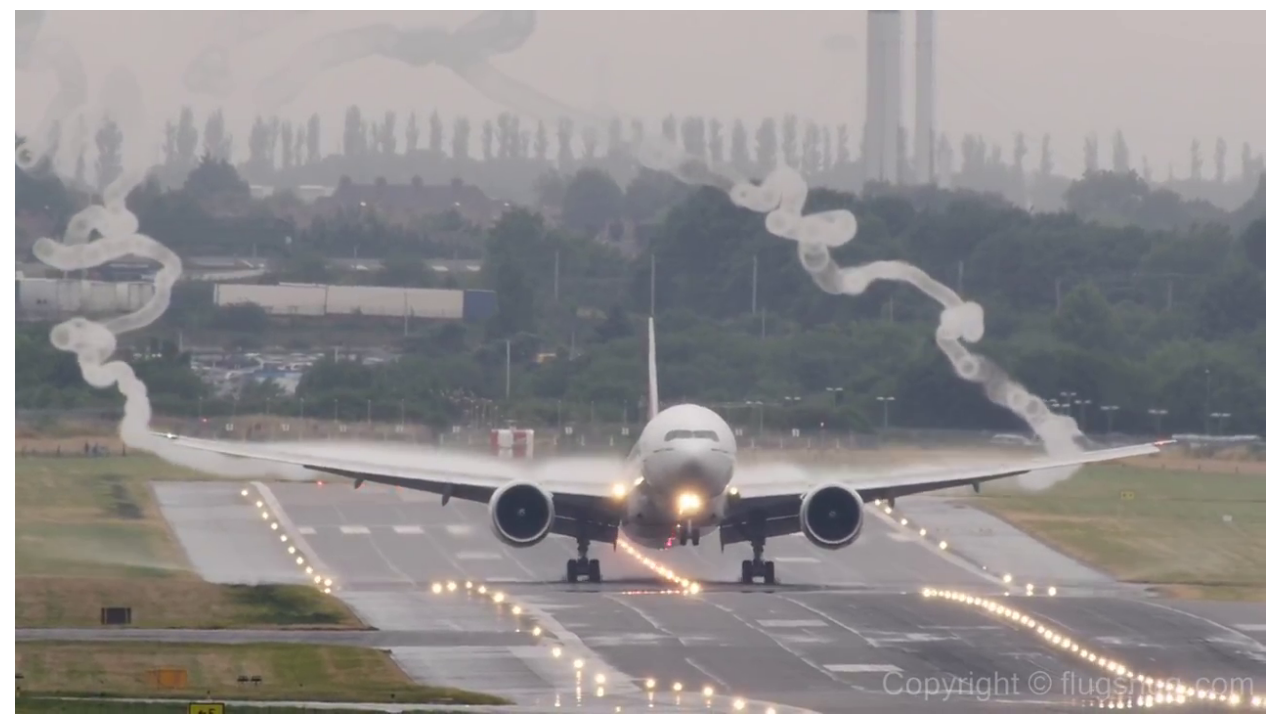

to characterize the wake properties of an aircraft flying through gusts with the already mentioned one-way coupling approach, and to use this to develop a more sophisticated methodology, a two-way coupling of an LES and a RANS code. This hybrid simulation shall provide a framework to simulate a flight through an atmospheric environment, the aircraft's response to atmospheric turbulence as well as the footprint of the wake to the atmosphere. This ambitious project will be based on the already established and partly validated one-way hybrid RANS/LES technique.

The unsteady behavior of an airfoil, which includes the flight through a gust, has been theoretically investigated since the early days of the last century. Wagner, Theodorsen and Küssner first developed exact analytical solutions for lift and drag in case of a 2-dimensional incompressible unsteady thin-airfoil flight for different forcing conditions, e.g. perturbation of angle of attack, gust response. A comprehensive summary can be found in Leishman (2006). Recent development in RANS simulation technologies have lead to a series of studies of gust encounters, e.g. Heinrich \& Reimer (2013), for real aircraft geometries. In this work the gust response of various gust functions was investigated with different simulation methods - lateral and vertical single gusts with a " 1 - cos" shape, but also series of periodically incoming gusts. It is of high interest to figure out the physical effect of different gust types on the wake vortices. However, this work investigates only one single case of a periodic lateral gust.

The paper presents the applied numerical methods first. The involved codes MGLET (LES) and TAU (RANS) are presented. The one-way coupling is described corresponding to Stephan et al., 2014, then the simulation setup and the boundary treatment are presented. The idea of the two-way coupling approach is described. Here we describe the coupling algorithm, as well as the hole cutting method used for the Chimera technique. We discuss the challenges of this approach and give a possible setup of an entire simulation consisting of three parts. Results from a single hybrid simulation using the one-way coupling approach are also presented.

\section{Numerical Method}

In this section we present the numerical concepts underlying the present study. Two hybrid RANS/LES methods are presented to simulate the flight through a gust. The first is based on a one-way coupling technique presented in Stephan et al. (2014). 


\section{CFD Codes}

MGLET - Multi-Grid Large Eddy Turbulence

The Large Eddy Simulation is performed using the incompressible Navier-Stokes code MGLET, developed at the Technical University of Munich, for solving the Navier-Stokes equations and the continuity equation, Manhart (2004)

$$
\begin{aligned}
\frac{\partial u_{i}}{\partial t}+\frac{\partial\left(u_{i} u_{j}\right)}{\partial x_{j}} & =-\frac{1}{\rho} \frac{\partial p^{\prime}}{\partial x_{i}}+\frac{\partial}{\partial x_{j}}\left(\left(\nu+\nu_{t}\right) 2 S_{i j}\right), \\
\frac{\partial u_{j}}{\partial x_{j}} & =0 .
\end{aligned}
$$

Here $u_{i}$ represents the velocity components in the three spatial directions $(i=1,2$, or 3$), S_{i j}=\left(\partial u_{i} / \partial x_{j}+\partial u_{j} / \partial x_{i}\right) / 2$ denotes the strain rate tensor and $p^{\prime}=p-p_{0}$ equals the pressure deviation from a reference state $p_{0}$. The kinematic viscosity is given as the sum of molecular viscosity $\nu$ and eddy viscosity $\nu_{t}$ determined by means of a Lagrangian dynamic sub-grid scale model, Meneveau et al. (1996). Eqn (1) and (2) are solved by a finite-volume approach, using a fourth-order finite-volume compact scheme, Hokpunna \& Manhart (2010). A split-interface algorithm is used for the parallelization of the tri-diagonal system, Hokpunna (2009), computing coefficients of the compact scheme. A third-order Runge-Kutta method is used for the time integration. The simulations are performed in parallel, using a domain decomposition approach.

\section{One-way coupling}

\section{Wake initialization}

We employ a wake initialization approach where a realistic aircraft wake is generated in an LES domain by sweeping a high-fidelity steady RANS flow field through the domain, which enables the simulation of the wake-vortex evolution from its generation until its final decay (Misaka et al., 2015). The simulations are performed for a long range aircraft model in high-lift configuration that has been used in ONERA's (Office National d'Etudes et de Recherche Aérospatiales) catapult facility during the European AWIATOR (Aircraft Wing with Advanced Technology Operation) project. The RANS flow field serves as a forcing term of the Navier-Stokes equations in the LES. This approach might be referred to as a fortified solution algorithm (Fujii, 1995), or a nudging technique used in data assimilation (Kalnay, 2003). The resulting velocity field in the aircraft vicinity consists of the weighted sum

$$
\mathbf{V}=f(y) \mathbf{V}_{\mathrm{LES}}+(1-f(y)) \mathbf{V}_{\mathrm{RANS}}
$$

of the LES and the RANS velocity field, see Figure 2, with a transition function

$$
f(y, \alpha, \beta)=\frac{1}{2}\left[\tanh \left[\alpha\left(\frac{y}{\beta}-\frac{\beta}{y}\right)\right]+1.0\right] .
$$

Here $\alpha$ and $\beta$ represent the slope and the maximum wall-distance of the transition region, for which we employ similar

Figure 2 Schematic of a weighting function for a combination of RANS and LES flow fields (Misaka et al., 2015).

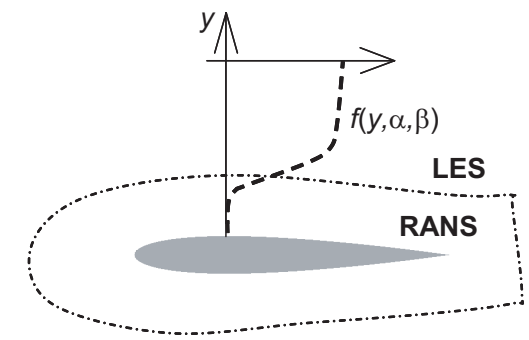

values as in Misaka et al. (2015). The mapping of the RANS flow field from an unstructured mesh to the structured grid of the LES solver is performed by a linear interpolation conducted only once before the wake initialization. First the steady RANS solution is mapped to a Cartesian grid, a so-called frame, which is then mapped to the LES grid and shifted for every time step. As the frame can only be mapped at discrete grid positions of the LES grid, an inclined glide path can not be realized by one fixed frame. For a glide path angle of 3.56 degrees this would imply on an equidistant grid that the frame has to be shifted 16 grid points horizontally and only one grid point vertically, leading to "steps" in the initialized wake. Therefore 16 frames have to be prepared in advance, interpolating the 
aircraft flow in between, leading to a smooth wake initialization. Note that the time step is determined by the grid spacing and the flight speed. For the explicit Runge-Kutta scheme, the Courant-Friedrichs-Levy (CFL) condition is an additional constrain on the time step. To achieve smaller CFL numbers, we halve the time step by doubling the number of interpolated frames. Hence, thirty-two frames of the RANS field are prepared in a pre-processing step and used alternately to achieve a realistically small aircraft descent angle. This requires additional memory, but it does not result in a significant increase of computational time for the simulation.

This hybrid method also enables us to study the effect of an ambient turbulent crosswind on the aircraft wake by initializing the wake to a pre-simulated turbulent wind field. In the present work stratification effects are not taken into account.

\section{Computational setting}

We employ a RANS flow field obtained by the DLR TAU-code, Keye (2011), from a steady compressible RANS simulation. An adaptive mesh refinement for wing-tip and flap-tip vortices, as well as the fuselage wake is employed. The flow conditions of the RANS simulation are the same as in ONERA's catapult experiment, i.e. chord based Reynolds number $\mathrm{Re}=5.2 \times 10^{5}$ (two orders of magnitude lower than the real aircraft flight), flight speed $U_{\infty}=$ $25 \mathrm{~m} / \mathrm{s}$, and a lift coefficient of $C_{L}=1.4$. The $1 / 27$ scaled model has a wingspan of $b=2.236 \mathrm{~m}$. We normalize quantities with the reference values for an elliptic load distribution (Gerz et al., 2002), initial circulation, vortex spacing, vortex descent velocity, characteristic time, vorticity unit,

$$
\Gamma_{0}=\frac{2 C_{L} U_{\infty} b}{\pi \Lambda}, \quad b_{0}=\frac{\pi}{4} b, \quad w_{0}=\frac{\Gamma_{0}}{2 \pi b_{0}}, \quad t_{0}=\frac{b_{0}}{w_{0}}, \quad \omega_{0}=\frac{1}{t_{0}},
$$

where $\Lambda=9.3$ is the wing aspect ratio. The resulting reference values for the normalization are $\Gamma_{0}=5.36 \mathrm{~m}^{2} / \mathrm{s}$ for circulation, $b_{0}=1.756 \mathrm{~m}$ for length, $w_{0}=0.49 \mathrm{~m} / \mathrm{s}$ for velocity, and $t_{0}=3.617 \mathrm{~s}$ for time. Normalized quantities are expressed in units of the reference values in (5) and are denoted by an asterisk. We set $t=0$ at the instant of the touchdown. The maximum tangential velocity of the wake vortices is around $10 \mathrm{~m} / \mathrm{s}$ (around $30 \mathrm{~m} / \mathrm{s}$ for a real aircraft).

\section{Ambient turbulent wind field}

In order to provide a realistic environment we establish a turbulent wind in a separate simulation. We simulate a turbulent half-channel flow with $\operatorname{Re}_{\tau}=3455$ applying a free-slip condition at the top of the domain, Moser et al. (1999). Prescribing initially a vertical wind profile following the universal logarithmic law and imposing a stream-wise pressure gradient of $d p / d y=1.2 \cdot 10^{-4} \mathrm{~N} / \mathrm{m}^{3}$, the wind flow is driven through the computational domain. We let the flow develop until characteristic wall streaks appear and an equilibrium between the pressure gradient and the wall friction is established. The averaged wind profile $\left\langle u^{+}\right\rangle$, see Figure 3 , converges to the typical half channel flow characteristics, Moser et al. (1999). The time-averaged stream-wise velocity of the wind at $b_{0}=\frac{\pi}{4} b$ above the ground is $w_{0}=0.49 \mathrm{~m} / \mathrm{s}$, where $w_{0}$ is the initial vortex descent speed. Let $\delta$ denote the channel half height and consider the following quantities as averaged in time. For the boundary layer approximation the Navier-Stokes equations yield the wall shear stress $\tau_{w}=-\delta \cdot \partial p / \partial x$, with constant pressure in wall-normal direction. The wall friction velocity is defined by $u_{\tau}=\left(\tau_{w} / \rho\right)^{1 / 2}$. This yields the normalized values $u^{+}=u / u_{\tau}, z^{+}=z u_{\tau} / \nu$ and an intrinsic Reynolds number $\operatorname{Re}_{\tau}=u_{\tau} \delta / \nu$. The boundary layer of the turbulent flow has three characteristic parts, the viscous sublayer, the transition layer and the logarithmic layer, see Figure 3.

\section{Reproduction of turbulent fluctuations}

From Czech et al. (2004) and Stephan et al. (2013) it is evident that the transfer of turbulent fluctuations in the wake to the LES field is important for the simulation of the wake evolution. In this work we use the approach employed by Misaka et al. (2015). As the eddy viscosity is modeled in the RANS computation it cannot be reproduced by the mapped velocity field. Hence, the modeled fluctuations have to be reproduced during the mapping process.

In a straightforward approach the velocity fluctuations are modeled as white noise in the RANS-LES transition region, controlling the magnitude by the proportional-integral $(\mathrm{PI})$ controller during the movement of the RANS field through the LES domain.

$$
\begin{aligned}
\mathbf{V}_{\mathrm{RANS}+\mathrm{WN}} & =\mathbf{V}_{\mathrm{RANS}}+K \mathbf{V}_{\mathrm{WN}} \\
K & =a_{1}\left(\bar{k}_{t, \mathrm{LES}}-k_{t, \mathrm{RANS}}\right) \\
& +a_{2} \int\left(\bar{k}_{t, \mathrm{LES}}-k_{t, \mathrm{RANS}}\right) d t
\end{aligned}
$$


Figure 3 Mean velocity profile characterizing the separately simulated turbulent wind.

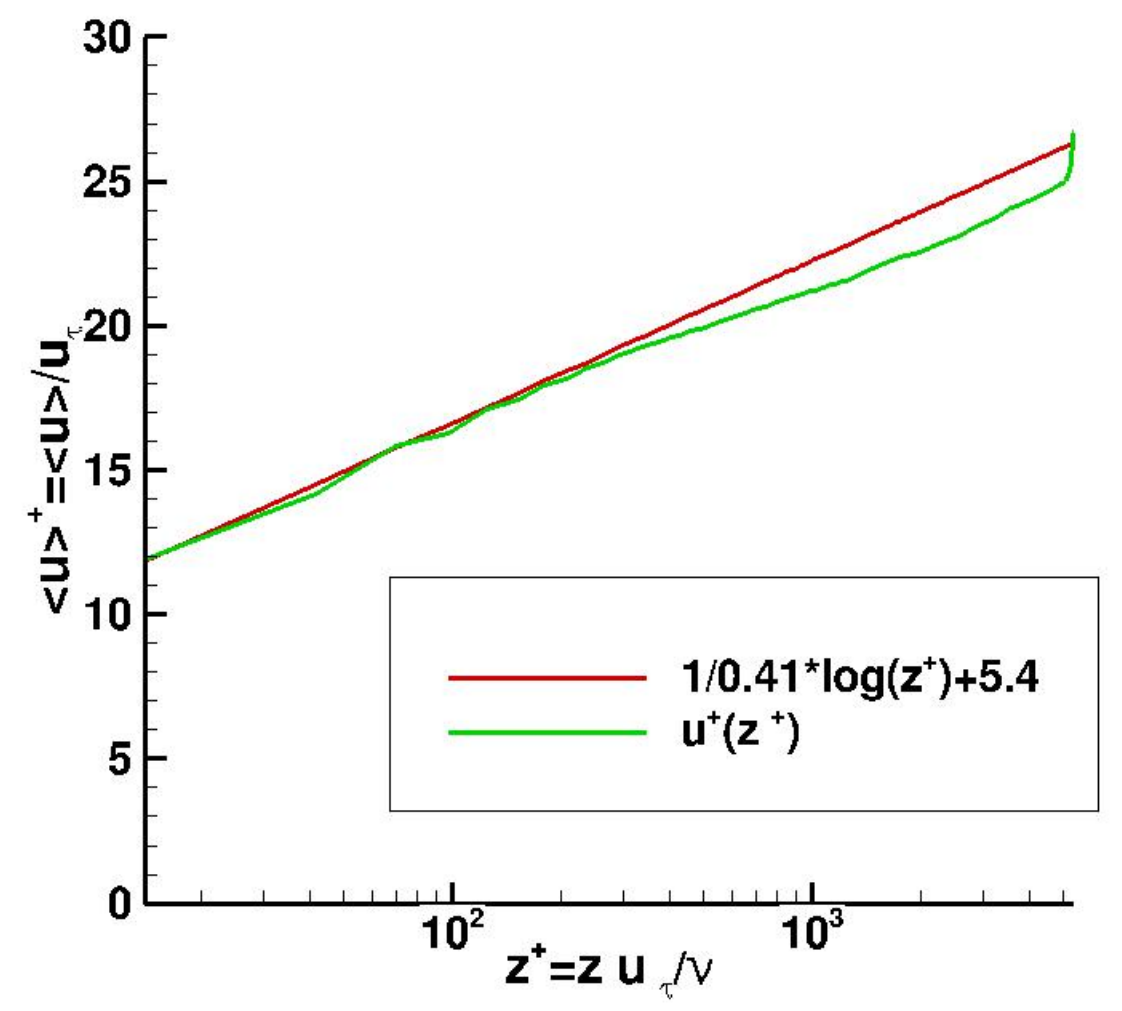

where $V_{\mathrm{WN}}$ denotes the white noise field and $K$ controls the magnitude. The constants are set to $a_{1}=40$ and $a_{2}=1.0$ in the simulations.

\section{Computational domain, approach and boundary treatment}

The hybrid simulation approach and the switch to pure LES (large arrow) is sketched in Figure 4. The first part of the simulations includes the hybrid RANS/LES wake initialization until touchdown, Figure 4 (a). The second part is a pure LES of the evolution of the aircraft wake, Figure 4 (b). We employ periodic boundary conditions in horizontal directions, a no-slip condition at the ground and a free-slip condition at the top. The aircraft starts in the back part of the domain, passes the boundary and approaches the ground. Because of the periodicity the aircraft is placed in front of the domain in Figure 4 (a). After touchdown the first slice of the domain is extended into the back part at the slope of the vortex and closed artificially to a horse shoe vortex, see Figure 4 (b). This procedure effectively minimizes disturbances generated at the starting point of the vortex initialization. Note that wake vortex linking due to Crow instability is frequently observed in cruise altitudes and may also occur in ground proximity. When the landing gear touches the ground the lift ceases quickly. Then the bound vortex, i.e. the circulation around the aircraft wings, and consequently the wake vortices are strongly reduced. We model the touchdown just by removing the RANS flow field forcing term from the simulation. In the LES domain we employ uniform mesh spacing for all three spatial directions, with a resolution of $d x^{*}=d y^{*}=d z^{*}=0.011$. Different dimensions of the complete computational domain are used for wake initialization and wake development, $23.3 b_{0}, 5.6 b_{0}$ to $11.6 b_{0}$, and $2.2 b_{0}$ in flight, spanwise and vertical directions, respectively, depending on the case, to capture the strong divergence of the vortices and the crosswind advection. The back part of the domain with a length of $5 b_{0}$ is where the aircraft motion starts and the subsequent reconnection of the vortex pair by a horseshoe vortex takes place. The aircraft passes the domain boundary at a height of $1.2 b_{0}$. At touchdown the tail wing is at $x^{*}=16.3$.

\section{Circulation modulation}

Küssner solved the problem of a sharp edge gust encounter with an up-wash velocity defined by the Heaviside function. With the principle of Duhamel we can find the lift response to an arbitrary gust, (Leishman, 2006, Chapter 
Figure 4 Schematic of an aircraft landing displaying the computational domain and the wake evolution (a) before touchdown, during wake initialization, (b) after touchdown with artificial vortex reconnection (from Stephan et al. (2014)).

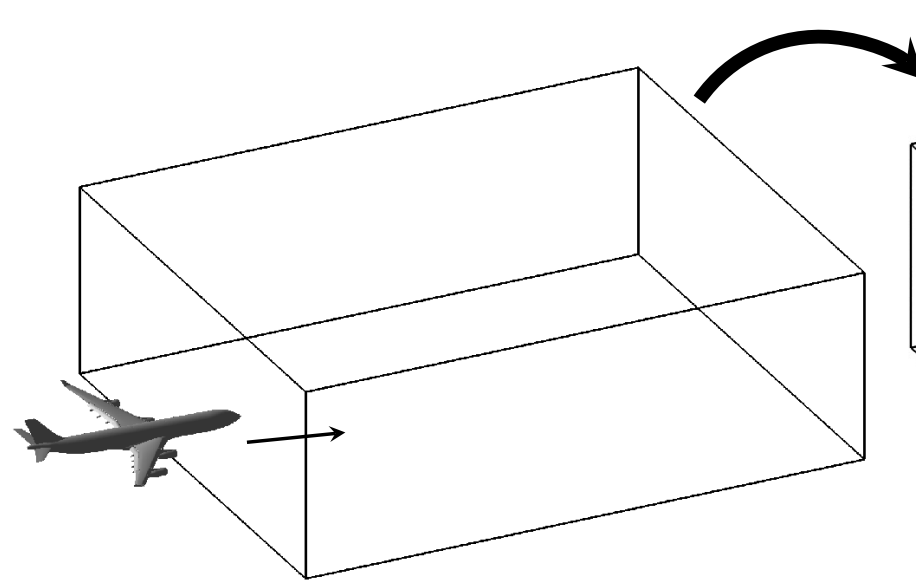

(a) Wake initialization until touchdown.

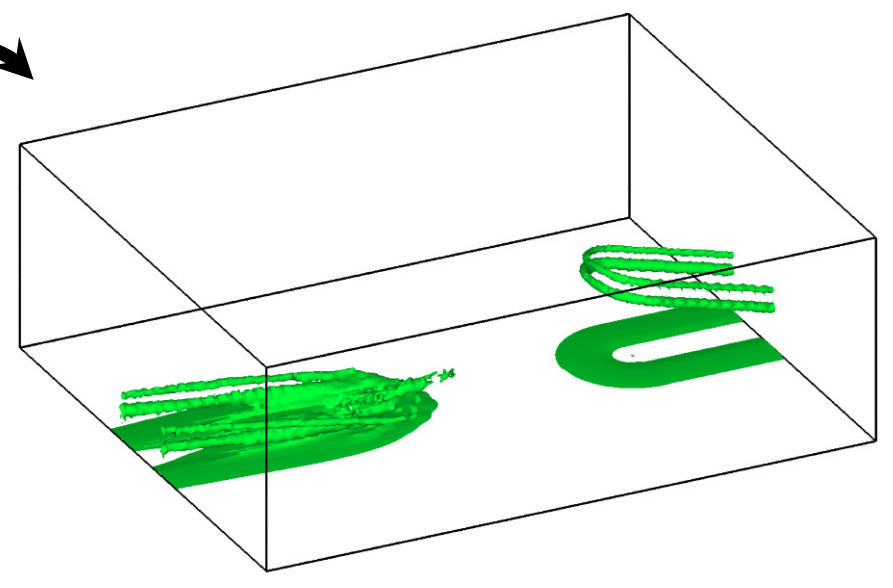

(b) LES, vortex decay after touchdown.

8). Here the Duhamel superposition using orthonormal series serves as a transformation from the gust signature to the aircraft response. For our investigation with the one-way coupling we focus on the aircraft's response and model the gust effect by a periodic modulation of the aircraft circulation. Heinrich \& Reimer (2013) shows that a lateral gust leads to an oscillation of the bank angle, which can be translated into an amplification and attenuation of the lift at the right and the left wing, respectively. This we translate to the circulation for the upwind and downwind vortex, by a sinusoidal modulation with an amplitude of $A=0.4 \Gamma_{0}$ and a wavelength of $\lambda=2.3 b_{0}$, which is in the order of the investigated cases in Heinrich \& Reimer (2013). Figure 5 depicts how the circulation $\Gamma$ of the wake vortex is modulated by a sinusoidal amplification

$$
\Gamma=\Gamma_{0}(1 \pm A \cos (2 \pi \cdot x / \lambda))
$$

modeling the effect of a periodical lateral gust, with "+" for the upwind vortex and "-" for the downwind. The wave crests are marked with a "+" and the wave troughs with a "-" in Figure 5. The wave crests descend faster, leading to a separation of the secondary vortex structures at the ground.

This straightforward approach is chosen because it is otherwise difficult to model a naturally occurring gust. We assume that for the vortex evolution the amplitude as well as the wavelength are the important parameters rather than the actual shape.

\section{Two-way coupling}

Modifying the pre-calculated RANS-solution of the aircraft's near-field to represent the effects of a gust on the wakevortex evolution is only a first approximation for the problem at hand. Clearly, the effects of the aircraft on the gust itself are not taken into account here. To better model these effects, a two-way-coupling is expected to be more suitable.

We are currently working on a two-way-coupling approach to enable us to simulate gusts, ambient turbulence and various other effects on the flow around the aircraft and the subsequent wake-vortex evolution. Here the coupling between the LES solver (MGLET) and the RANS solver (TAU) is based on the Chimera technique for overlapping meshes. The details of the coupling implementation will be presented in the following sections. Simulation results will be presented in a later publication.

\section{Coupling Algorithm}

At the core of the coupling algorithm lies the Chimera technique - first introduced by BENEK et al. (1983) and successfully applied by numerous authors Reuß et al. (2012) Eyi et al. (1996), = Sankaran et al. (2011). The main idea behind is to use independent computational meshes that exchange flow variables at the interfaces between them.

A classical example from aerodynamics is the simulation of an aerodynamic foil with a flap in different configurations. Instead of creating a new mesh for the combination of airfoil and flap, Chimera makes it possible to employ two separate meshes - one around the airfoil and another one around the flap. In order to run simulations at different flap 
Figure 5 Periodic circulation modulation of the aircraft wake, aircraft exposed to a lateral periodic gust. Ground surface colored by voricity magnitude. Iso-surface of vorticity represents the wake vortices.
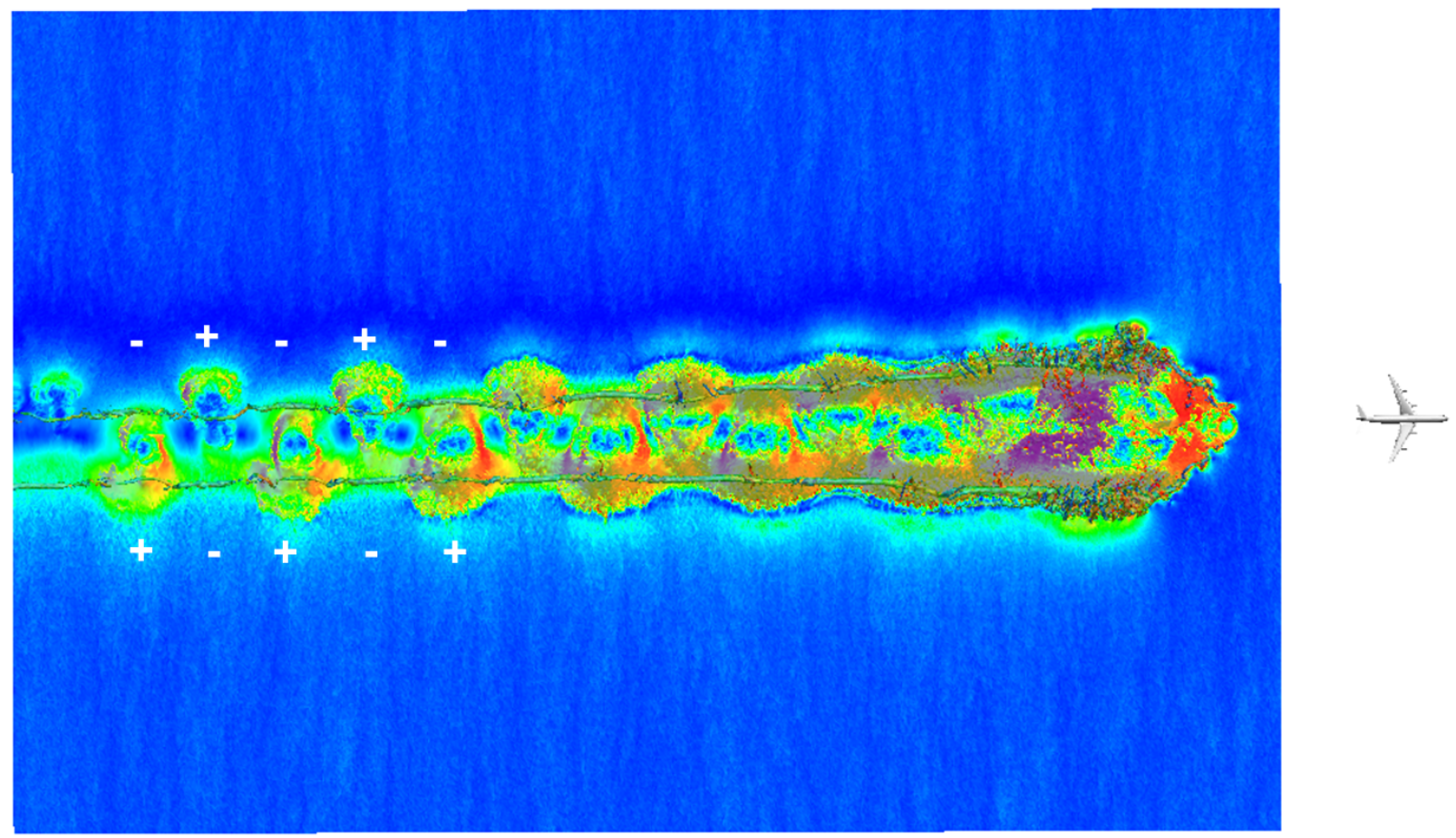

angles only the flap component mesh has to be moved and no remeshing is needed. Chimera is therefore particularly attractive for simulations with relative motions.

In the present case, the LES domain represents a chunk of the atmosphere (fixed in space) and the RANS domain spans the near-field of the aircraft. Similar to the one-way methodology, the second domain is moving through the first one and is hereby inducing turbulent vortical structures, the evolution of which we are interested in. However, this time both the LES and the RANS domain exchange flow values at the interfaces between them. It is therefore important to guarantee that there is enough overlap between both meshes at the coupling interface. Otherwise an interpolation source point could coincide with an interpolation target point which will inevitably lead to false results.

In general, there are two approaches available for coupling flow solvers. They can be either coupled directly or through a separate application (coupling module). The implementation of the first approach is often more difficult, since the needed search and interpolation procedures have to be implemented separately for each flow solver. Additionally, for Massage Passing Interface (MPI) applications the MPI_COMM_WORLD communicator has to span both applications in order to facilitate the communication between the solvers. Separate MPI communicators have to be used within each flow solver, which increases the implementation effort.

We opted for the second approach - using a coupling application. Here each flow solver sends its complete grid to the coupling module, which is then responsible for the search and interpolation procedures. The coupling module that we used was developed at the DLR's Institute of Aerodynamics and Flow Technology in Brunswick Schwarz et al. (2010). In order to eliminate the problem with the multiple MPI communicators mentioned above, it employs socket communication between coupling module and solver. Within the coupling module itself, MPI communication is used.

Each flow solver only has to identify the boundary points where it needs interpolated values from the other solver, send the indices of those points to the coupling module and later receive the values of the requested points. Additionally, each solver has to provide the flow variables for the interpolation source points needed by the coupling module to calculate the interpolated values for the other solver(s). This is made possible by sending the complete mesh from each solver to the coupling module.

To optimize the socket communication when running the coupled simulation on high-performance computing (HPC) clusters, it is being restricted within a single node. This means that on every computing node the same amount of solver and coupling module processes are being started - one coupling module process for every solver 
process. The socket communication only takes place within a node, between solver and coupling module, which drastically improves communication speed.

\section{Hole cutting}

Hole cutting is an integral part of the Chimera technique. The hole defines the unity of cells where no solution has to be calculated, i.e. cells located within a solid body. Additionally, the fringe points of the hole define interpolation target points where flow variables will be interpolated from the other solver. These points represent an artificial boundary in the domain.

There are many hole cutting methods for structured and unstructured meshes, Chan \& Pandya (2015), Vuik et al. (2000). In MGLET we employ the fast triangle-box intersection test by Akenine-Möller (2005) to identify the cells which intersect the hole surface. The hole geometry is read from an STL-file, where surfaces are represented as triangles. Since more than one interpolation point layers are needed at the interface in order to guarantee a full computational stencil for the boundary points, these extra points have to be identified in a second step. First, the points within the hole are identified. This is done by specifying parts of the domain as fluid cells at all times (e.g. a corner of the domain where no solid body will be present at any time), iteratively marching to their neighbors and marking those as fluid cells if they haven't been marked as surface cells in the previous step. At the end of this iterative process all fluid cells will be identified. Additional interpolation point layers can be added in a similar manner, this time starting the iteration from the marked surface boundary points and marking only adjacent fluid cells as interpolation points.

\section{Coupling challenges}

In segregated hybrid RANS-LES simulations, one of the biggest challenges is the generation of turbulent structures at parts of the interface where these are convected into the LES domain. This is needed, since Large Eddy Simulations resolve part of the turbulent spectrum, whereas RANS simulations only deliver statistical mean values. Several methodologies exist with synthetic turbulence generation being the most promising. The idea behind it is to generate turbulent flow structures at the RANS-LES-interface, based on one or more statistical turbulence quantities delivered by the RANS simulation.

The coupling of the pressure between the compressible RANS and the incompressible LES solvers poses a second challenge. The fluid incompressibility in the LES domain implies that acoustic waves travel at infinite speeds, which is not the case in the compressible RANS domain. The interface between both solvers has to take those differences in the mathematical models into account. We are currently working on those challenges.

\section{Setup}

The computational setup for our future simulations consists of two code instances of the RANS solver TAU (referred to as TAU1 and TAU2) and one instance of the LES solver MGLET. Figure 6 shows the corresponding computational domains. TAU1's domains consist of two Cartesian meshes (in blue) that are needed for the start and the end of the unsteady simulation. TAU2's domain is represented by an unstructured hybrid mesh around the wing (in black,

Figure 6 TAU's cartesian domain (in blue), MGLET's Cartesian domain (in red) and TAU's unstructured domain (in black) at the beginning of the unsteady simulation

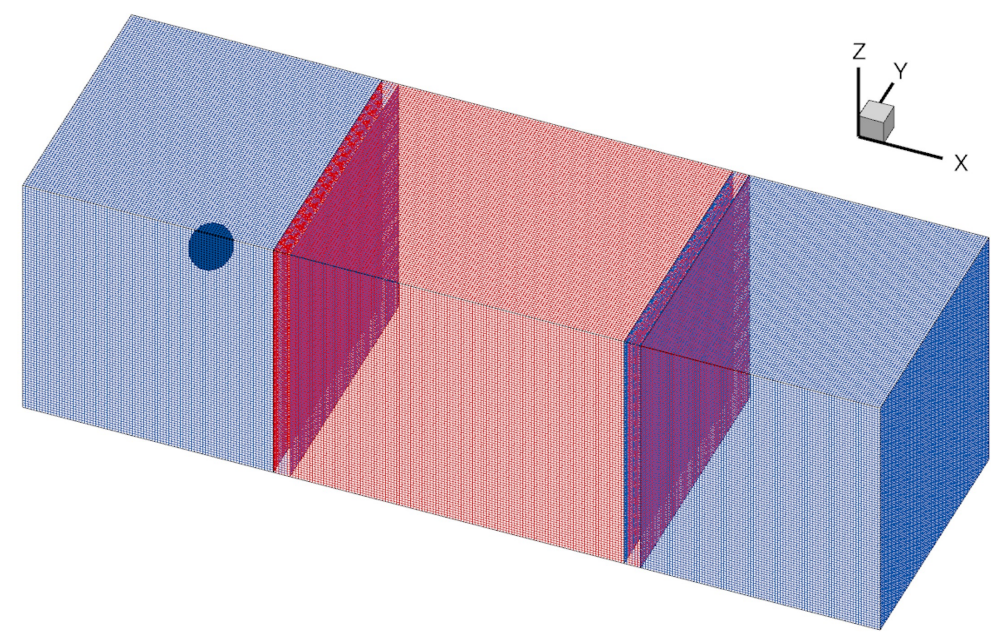


detailed view in Figure 7). For the unsteady simulation, this domain will move into MGLET's domain. Note that we use a 3D wing geometry (ONERA's M6 wing) for our test simulations, which we mirrored to form a full wing. Later it will be replaced by a full aircraft geometry.

Figure 7 TAU's domain of the wing's near-field and hole geometry (in red)

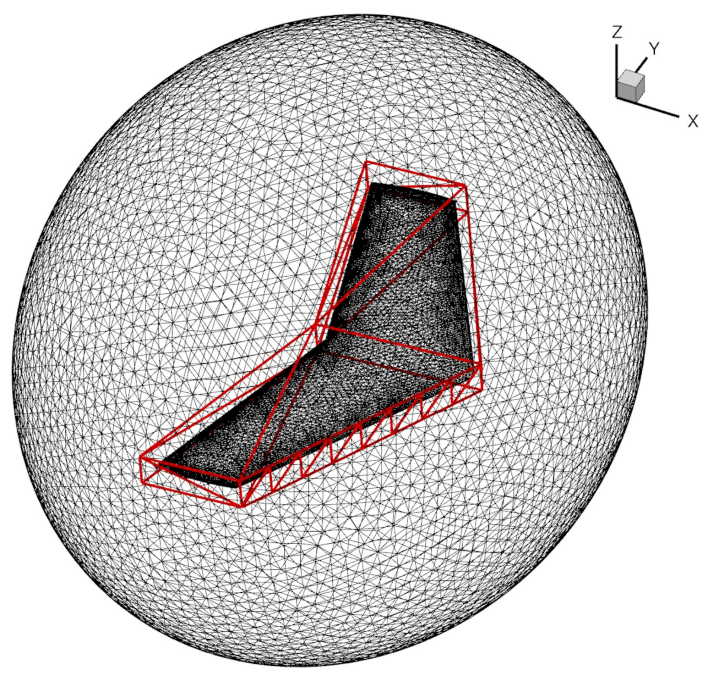

Before starting the coupled unsteady simulation, a steady state solution has to be found for domain TAU2. Since this domain only spans the aircraft's near field, we need to extend it by coupling it to one of the blocks of domain TAU1 that surrounds it. TAU1's boundaries are placed far enough (around 70 chord lengths) from the aircraft in order for a far field boundary condition to be correctly employed. A hole is defined within domain TAU2 (see Figure 7) around the aircraft and all fluid variables at this hole's surface are communicated to domain TAU1. TAU2 gets values from TAU1 at its outer boundary. This coupling is realized through the coupling module described in the previous sections.

In the next step the unsteady coupled simulation is started. Domains TAU1 and MGLET are stationary and domain TAU2 is moving (here in positive $x$ direction), see Figure 8. It's movement is prescribed in a Python script that communicates it to MGLET through a file. TAU gets the movement information directly via static variables in Python, since TAU's internal functions were pythonized using the interface compiler SWIG and can be invoked directly in Python, which is particularly convenient for coupled simulations. For the unsteady simulation, MGLET's left and

Figure 8 TAU's cartesian domain (in blue), MGLET's Cartesian domain (in red) and TAU's unstructured domain (in black) after the near-field domain has entered the MGLET domain

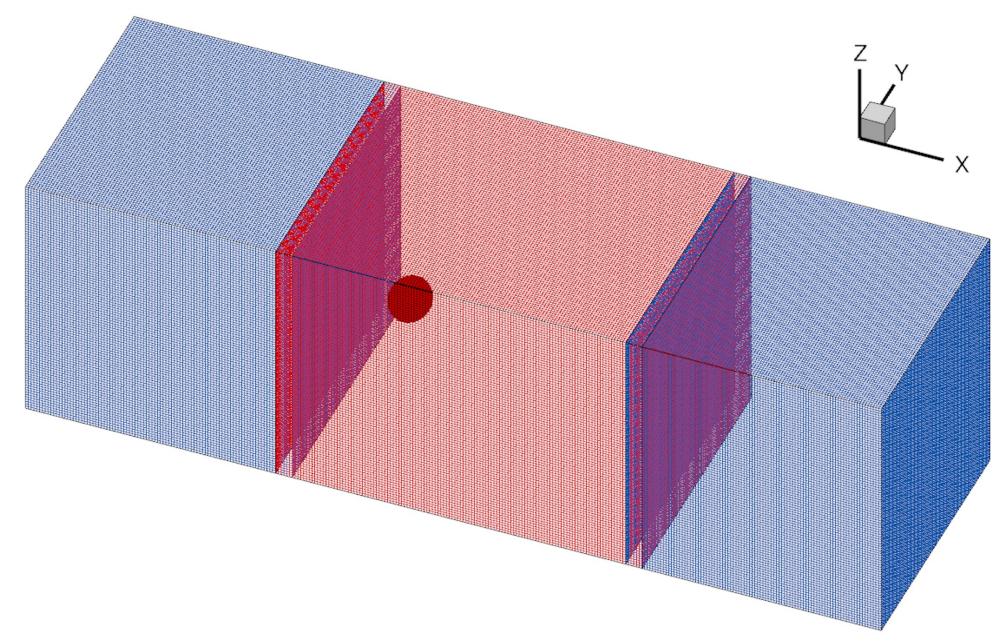

right boundaries are coupled with the Cartesian TAU domains, so that velocity and pressure disturbances can enter MGLET's domain long before domain TAU2 reaches this coupling boundary.

The second TAU domain on the right side of MGLET's domain is necessary, in order to allow domain TAU2 to exit the LES domain. After that all TAU simulations can be stopped and the vortex evolution can be further simulated 
in MGLET, employing periodic boundary conditions in lateral and vertical directions, whereas the quasi periodic approach described in Sec. is applied to the flight direction.

\section{Results of the one-way coupling}

\section{Flow field}

The flow field during the vortex initialization phase results in a complex interaction of the aircraft wake system and the crosswind.

The crosswind situation, where we apply a uniform vortex initialization technique, is discussed in Stephan et al. (2013).

Crosswinds induce vorticity at the ground, which has opposite sign as the vorticity layer induced by the up-wind vortex and the same sign as the vorticity layer induced by the downwind vortex (Figure 9). Thus, the crosswind vorticity supports the formation of the downwind vorticity layer and attenuates the upwind vorticity layer. As a consequence, vorticity layers generated by the wake vortices become unequally strong and the upwind and downwind vortices behave asymmetrically. The magnitudes of the wake-vortex induced vorticity layers are growing, leading eventually to separation and the generation of counter-rotating secondary vortices, first at the downwind and then at the upwind vortex. Then the secondary vortices rebound and interact with the primary vortices.

Figure 9 Sketch of wake vortex flow with crosswind.

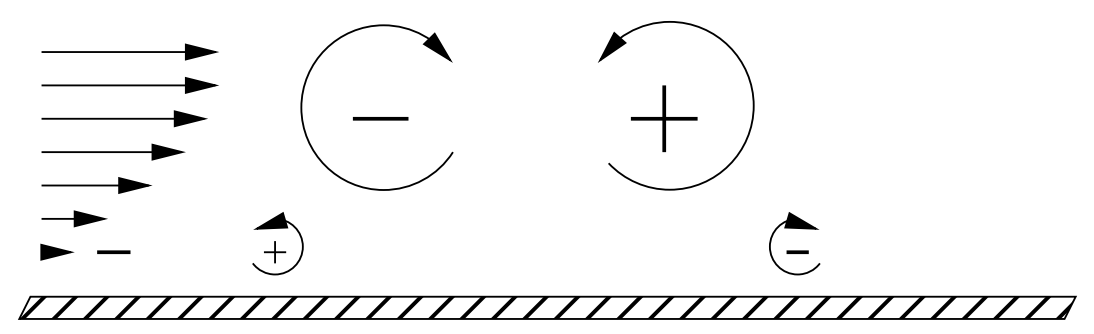

The secondary vorticity layer can be observed in Figure 10. As explained it is more pronounced for the downwind vortex. The intensity increases closer to the point of touchdown. Due to the circulation modulation the intensity increases not uniformly, but in waves. The wake mainly consists of the co-rotating flap tip and wing tip vortices. The roll-up process is disturbed by the circulation modulation, Figure 10 (b), leading finally to a wave pattern in the fully rolled-up vortices, see Figure 10 (c), which is more pronounced for the downwind vortex. After touchdown, disturbances, the so-called end effects appear, propagating against the flight direction. These end effects were investigated in detail in Stephan et al. (2014). They mainly consist of two phenomena, the ground effect and the touchdown effect, where the lift of the aircraft suddenly reduces and pressure as well as circulation disturbances appear. The secondary vorticity is more strongly pronounced where the primary circulation is large and close to the ground Figure 10 (d). Here, strong secondary vortex structures are formed, wrapping around the primary vortices. This leads finally to vortex deformation at the same scale as the circulation is modulated. After touchdown we observe the vortex evolution in Figure 11. The circulation decay is triggered by the vortex irregularities. The initialized secondary vortex structures determine nuclei of vortex decay. The downwind vortex is advected with the wind, whereas the upwind vortex remains in the proximity of the runway. Close to the touchdown zone this process is more pronounced, leading to a strong divergence of the vortices. The instabilities of the primary vortices remain somewhat stable. Only in the proximity of the touchdown zone we observe vortex linking with the ground, Figure 11 (e)-(f). There the vortices mount up to a height of approximately $b_{0}$. This qualitative investigation of the vortex evolution already reveals that the upwind vortex is decaying faster than the downwind vortex. This situation has never been observed in wake vortex simulations in a crosswind situation Holzäpfel et al. (2016) as well as Bricteux et al. (2016) and originates in the interaction of the modulated vorticity with the crosswind.

\section{Circulation, Trajectories}

Figure 12 (a), (b) depicts the circulation evolution of the gust effect of both the upwind as well as the downwind primary vortex, starting at the instant of touchdown. Figure 12 (c), (d) depicts the same situation without the gust effect. As could be observed before the vortex circulation increases in the proximity of the touchdown zone, Stephan et al. (2014), the circulation modulation is clearly visible. However, parts of strong and weak circulation are already balancing each other during the initialization phase. The decay process is not uniform, but reveals the complex interaction of end effects, ground, wind and modulated vortex. The end effects are clearly visible propagating in 
time, however they are suppressed for the downwind vortex by the circulation instabilities, which leads to a relative stability. The upwind vortex decays much faster, particularly the interaction between end effect and vortex modulation intensifies the vortex decay. For the downwind vortex the initial circulation modulations almost level out.

The vortex height above ground $z$ is depicted in Figure 13. Depending on the initialization height $z_{0}^{*}$ the trajectories differ, due to three dimensional large scale variations in time. This effect is more pronounced for the downwind vortex. In average the classical rebound behavior is reproduced, but not slice wise, where we observe strong vortex rebound close to the touchdown zone as well as vortex linking with the ground. In most of the slices a differently pronounced vortex descend is observed after the initial rebound. Please note that after $t^{*}=3$ the vortex deformation growth is too large and the vortices link with the domain boundaries. Therefore at $z_{0}^{*}=0.5$ as well as $z_{0}^{*}=1.1$ the plots show unphysical behavior for the downwind vortex at $3 t_{0}$.

\section{Conclusion}

The unsteady aircraft landing and the subsequent wake vortex evolution is investigated in the present work. Two different concepts are presented as possible ways to tackle this problem. The one-way coupling as well as the concept of the two-way coupling are presented. The already matured one-way coupled hybrid RANS/LES simulation technique can be used to develop and assess the much more sophisticated two-way coupling approach. The oneway coupling simulation with a sinusoidal modulation of the vortex circulation is presented, modeling the aircraft's response of a flight through a lateral periodic gust. The two-way coupling still lacks the right way to communicate turbulent structures at the interfaces.

The analysis of the complex flow field shows an instantaneous deformation of the primary vortices, which can be observed in reality. The circulation modulation leads to several unexpected results. No vortex linking except for the touchdown linking occurs. The circulation irregularities balance and level out. The initial deformation is not accelerating but keeps the vortices stable, however, this may depend on the wave length of the circulation modulation. The upwind vortex is even more stable and decays faster than the downwind vortex.

Finally, one can deduce that the physics of the unsteady aircraft landing is very challenging, leading to highly complex flows and cannot be explained by a single simulation. Specific questions have to be formulated, leading to specific gust and wind initializations. These are still pending for a comprehensive understanding of real wake vortex behavior under complex realistic landing conditions.

\section{Acknowledgments}

We would like to thank Stefan Melber (Institut für Aerodynamik und Strömungstechnik, DLR-Braunschweig) for providing the RANS data of the AWIATOR long range aircraft model. We also acknowledge Airbus for the allowance to use it in our simulations. We thank Michael Manhart and Florian Schwertfirm for the provision of the original version of the LES code MGLET. The CPU time on the HPC Cluster SuperMUC, provided by the Leibniz Supercomputing Centre (LRZ), is greatly acknowledged.

\section{References}

Akenine-Möller, T. (2005), Fast 3D triangle-box overlap testing. ACM SIGGRAPH 2005 Courses on - SIGGRAPH '05, pp. 8. ACM Press, New York, New York, USA. doi:10.1145/1198555.1198747.

BENEK, J., STEGER, J., DOUGHERTY, F. (1983), A flexible grid embedding technique with application to the Euler equations. 6th Computational Fluid Dynamics Conference Danvers. American Institute of Aeronautics and Astronautics, Reston, Virigina. doi:10.2514/6.1983-1944.

Bricteux, L., Duponcheel, M., De Visscher, I., Winckelmans, G. (2016), LES investigation of the transport and decay of various-strengths wake vortices in ground effect and subjected to a turbulent crosswind. Physics of Fluids, Vol. 28, no. 065105.

Chan, W. M., Pandya, S. A. (2015), Advances in Distance-Based Hole Cuts on Overset Grids. 22nd AlAA Computational Fluid Dynamics Conference, pp. 1-19. American Institute of Aeronautics and Astronautics, Reston, Virginia. ISBN 978-1-62410-366-7. doi:10.2514/6.2015-3425.

Czech, M. J., Miller, G. D., Crouch, J. D., Strelets, M. (2004), Near-field Evolution of Trailing Vortices Behind Aircraft with Flaps Deployed. AIAA Paper, Vol. 2149.

Eyi, S., Lee, K. D., Rogers, S. E., Kwak, D. (1996), High-lift design optimization using Navier-Stokes equations. Journal of Aircraft, Vol. 33, No. 3, pp. 499-504. ISSN 0021-8669. doi:10.2514/3.46972. 
Fujii, K. (1995), Unified Zonal Method Based on the Fortified Solution Algori. Journal of Computational Physics, Vol. 118, pp. 92-108.

Georges, L., Geuzaine, P., Duponchel, M., Bricteux, L., Lonfils, T., Winckelmans, G., Giovannini, A. (2005), Technical Report 3.1.1-3, LES of two-vortex system in ground effect with and without wind. Techn. Ber., Université catholique de Louvain (UCL), Institut de Mécanique des Fluides de Toulouse(IMFT).

Gerz, T., Holzäpfel, F., Darracq, D. (2002), Commercial aircraft wake vortices. Progress in Aerospace Sciences, Vol. 38, No. 3, pp. 181-208.

Heinrich, R., Reimer, L. (2013), Comparison of different approaches for gust modeling in the CFD Code TAU. International Forum on Aeroelasticity \& Structural Dynamics 2013.

Hokpunna, A. (2009), Compact Fourth-order Scheme for Numerical Simulations of Navier-Stokes Equations. PhD Thesis, Technische Universität München (Germany).

Hokpunna, A., Manhart, M. (2010), Compact Fourth-order Finite Volume Method for Numerical Solutions of navierStokes Equations on Staggered Grids. Journal of Computational Physics, Vol. 229, No. 20, pp. 7545-7570.

Holzäpfel, F., Gerz, T., Baumann, R. (2001), The Turbulent Decay of Trailing Vortex Pairs in Stably Stratified Environments. Aerospace Science and Technology, Vol. 5, No. 2, pp. 95-108.

Holzäpfel, F., Tchipev, N., Stephan, A. (2016), Wind Impact on Single Vortices and Counterrotating Vortex Pairs in Ground Proximity. Flow, Turbulence and Combustion., pp. 1-20. doi:10.1007/s10494-016-9729-2 ISSN 1386-6184.

Kalnay, E. (2003), Atmospheric Modeling, Data Assimilation and Predictability. Cambridge University Press.

Keye, S. (2011), Fluid-Structure Coupled Analysis of a Transport Aircraft and Flight-Test Validation. Journal of Aircraft, Vol. 48, No. 2, pp. 381-390.

Leishman, J. G. (2006), Principles of Helicopter Aerodynamics. Cambridge University Press.

Manhart, M. (2004), A Zonal Grid Algorithm for DNS of Turbulent Boundary Layer. Computer and Fluids, Vol. 33, No. 3, pp. 435-461.

Meneveau, C., Lund, T. S., Cabot, W. H. (1996), A Lagrangian dynamic subgrid-scale model of turbulence. Journal of Fluid Mechanics, Vol. 319, pp. 353-385.

Misaka, T., Holzäpfel, F., Gerz, T. (2015), Large-Eddy Simulation of Aircraft Wake Evolution from Roll-Up until Vortex Decay. AIAA Journal, Vol. 53, pp. 2646-2670. doi:10.2514/1.J053671.

Misaka, T., Holzäpfel, F., Hennemann, I. Gerz, T., Manhart, M., Schwertfirm, F. (2012), Vortex bursting and tracer transport of a counter-rotating vortex pair. Physics of Fluids, Vol. 24, pp. (025104-1) - (025104-21).

Moser, R. D., Kim, J., Mansour, N. (1999), Direct numerical simulation of turbulent channel flow up to $R e=590$. Physics of Fluids, Vol. Vol. 11, 4, pp. 943-945.

Proctor, F. H., Hamilton, D. W., Han, J. (2000), Wake Vortex Transport and Decay in Ground Effect: Vortex Linking with the Ground. AIAA, 2000-0757, 38th Aerospace Sciences Meeting \& Exhibit, Reno.

Reuß, S., Wolf, C., Knopp, T., Raichle, A., Schwamborn, D. (2012), Chimera technique for transporting disturbances. International Journal for Numerical Methods in Fluids, Vol. 70, No. 12, pp. 1558-1572. ISSN 02712091 . doi: 10.1002/fld.3642.

Sankaran, V., Wissink, A., Datta, A., Sitaraman, J., Potsdam, M., Jayaraman, B., Katz, A., Kamkar, S., Roget, B., Mavriplis, D., Saberi, H., Chen, W.-B., Johnson, W., Strawn, R. (2011), Overview of the Helios Version 2.0 Computational Platform for Rotorcraft Simulations. 49th AIAA Aerospace Sciences Meeting including the New Horizons Forum and Aerospace Exposition, pp. 1-22. American Institute of Aeronautics and Astronautics, Reston, Virigina. ISBN 978-1-60086-950-1. doi:10.2514/6.2011-1105.

Schwarz, T., Spiering, F., Kroll, N. (2010), Grid coupling by means of Chimera interpolation techniques Overset grid approach. pp. 1-12.

Spalart, P. (1998), Airplane trailing vortices. Annual Review of Fluid Mechanics, Vol. 30(1), pp. 107-138. 
Stephan, A., Holzäpfel, F., Misaka, T. (2013), Aircraft Wake Vortex Decay in Ground Proximity - Physical Mechanisms and Artificial Enhancement. Journal of Aircraft.

Stephan, A., Holzäpfel, F., Misaka, T. (2014), Hybrid simulation of wake-vortex evolution during landing on flat terrain and with plate line. International Journal of Heat and Fluid Flow, Vol. 49, pp. 18-27.

Vuik, C., Saghir, A., Boerstoel, G. P. (2000), A fully automated Chimera methodology for multiple moving body problems. International Journal for Numerical Methods in Fluids, Vol. 33, No. 7, pp. 919-938. ISSN 02712091. doi: 10.1002/1097-0363(20000815)33:7<919::AID-FLD944>3.0.CO;2-G. 
Figure 10 One-way hybrid simulation of an aircraft landing, vorticity iso-surface showing wing-tip and flap-tip vortex, vorticity magnitude at the ground showing wind and secondary vorticity. Wind direction from right to left.
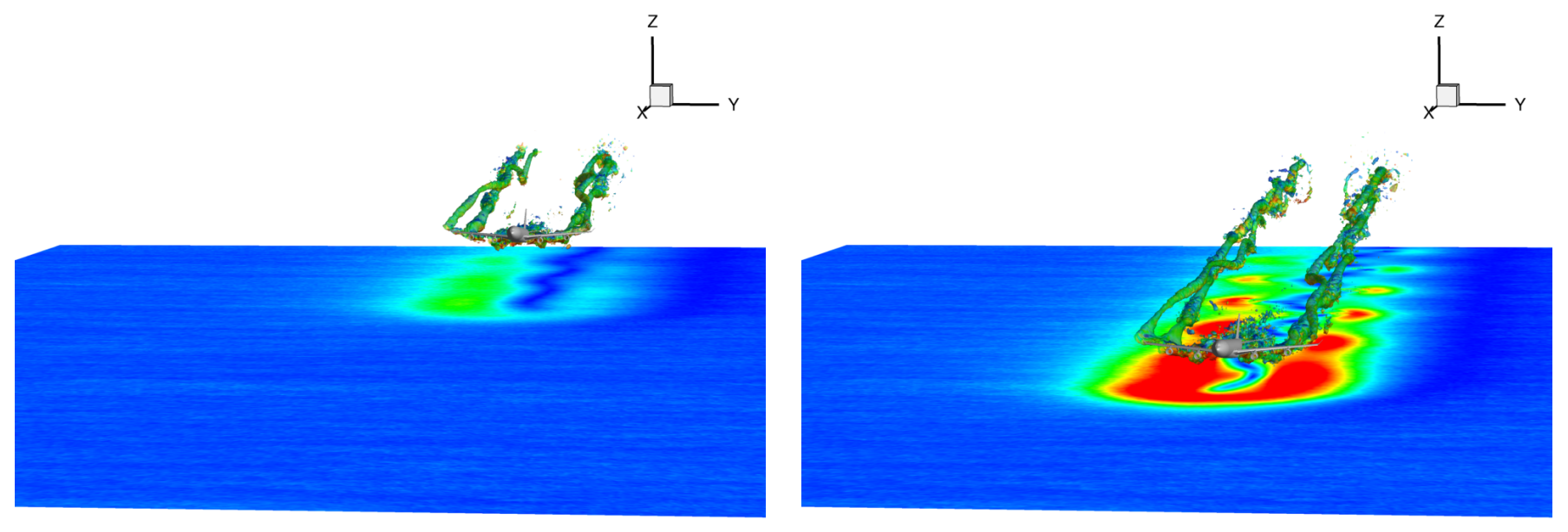

(a) $t^{*}=-0.18$

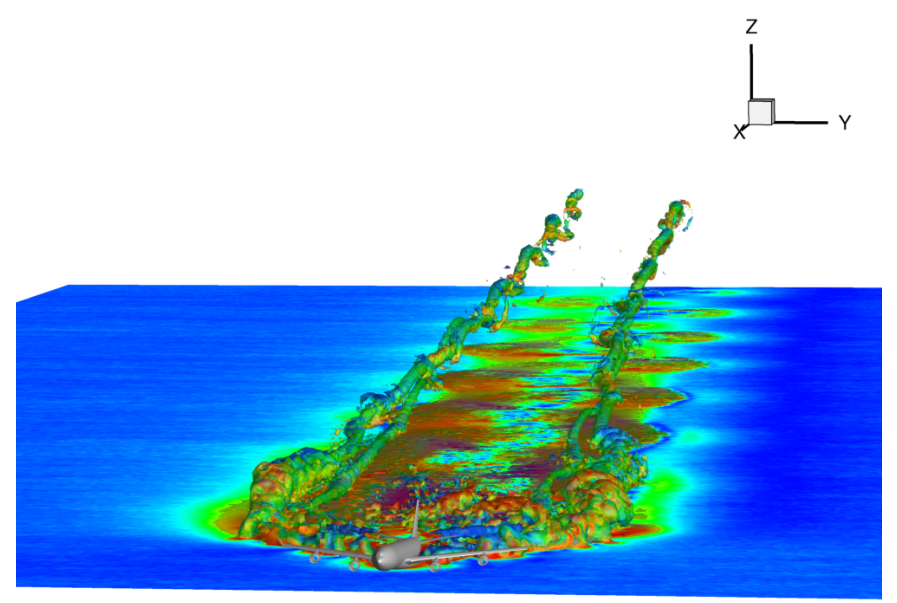

(c) $t^{*}=0.02$ (b) $t^{*}=-0.07$

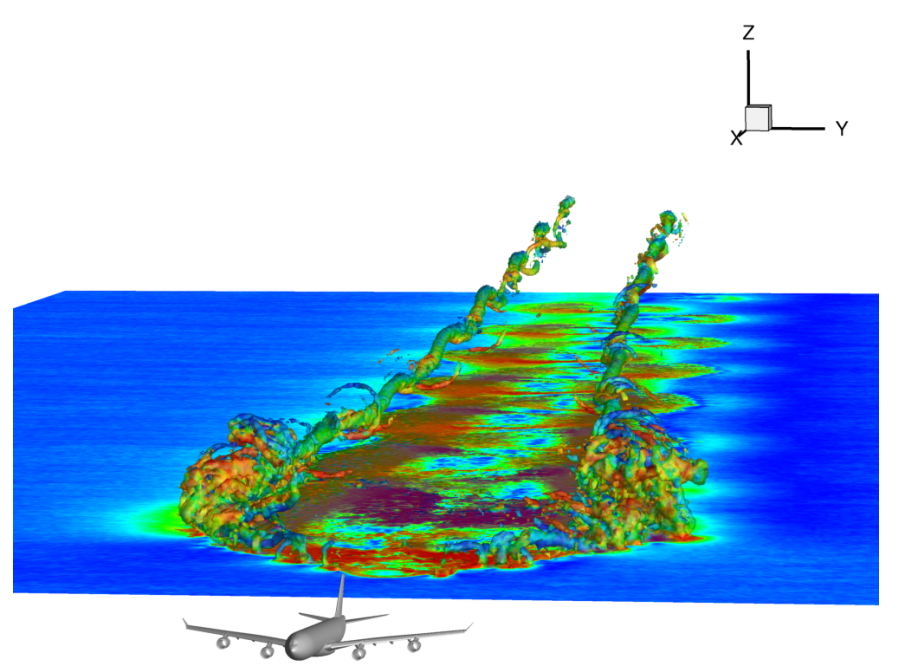


Figure 11 Wake vortex evolution after touchdown, vortex decay with end effects, ground slice colored by vorticity magnitude, vorticity iso-surface showing aircraft wake.

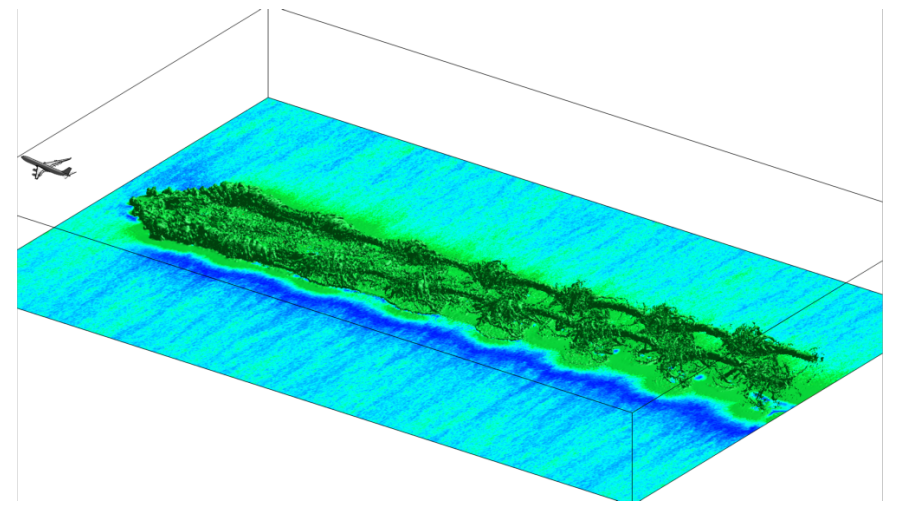

(a) $t^{*}=0.07$

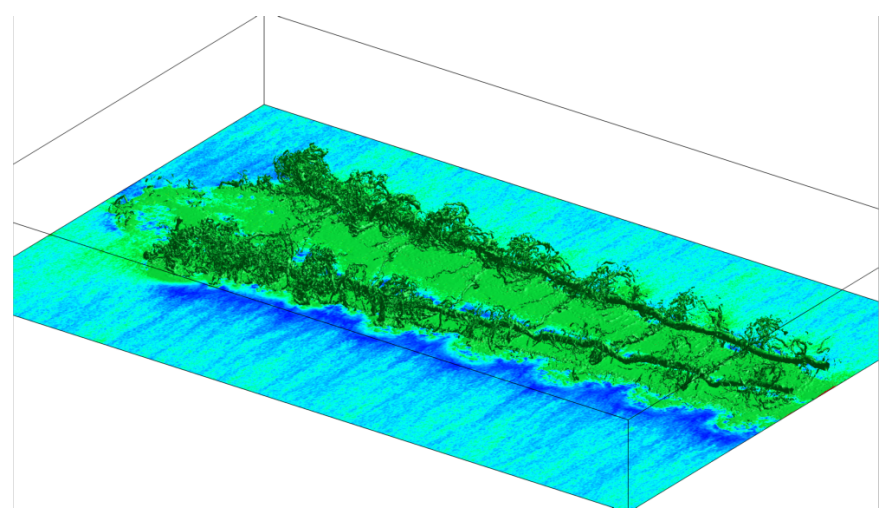

(c) $t^{*}=0.6$

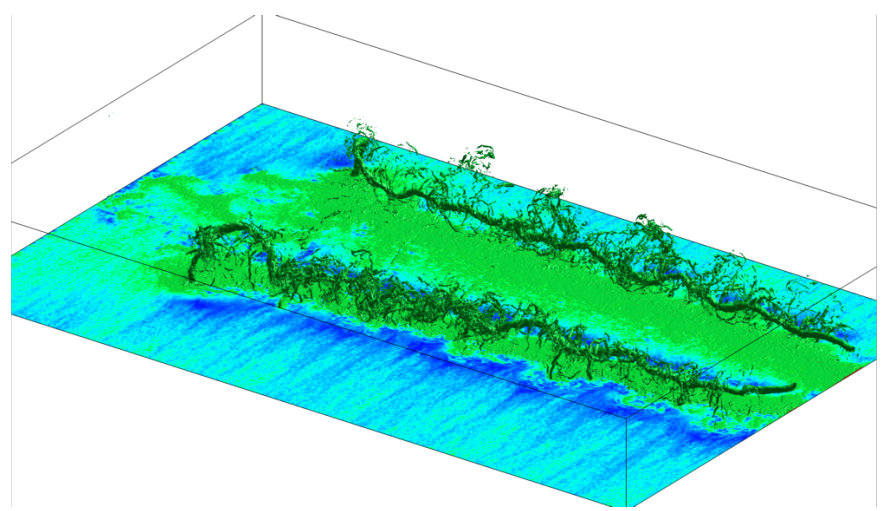

(e) $t^{*}=1.3$

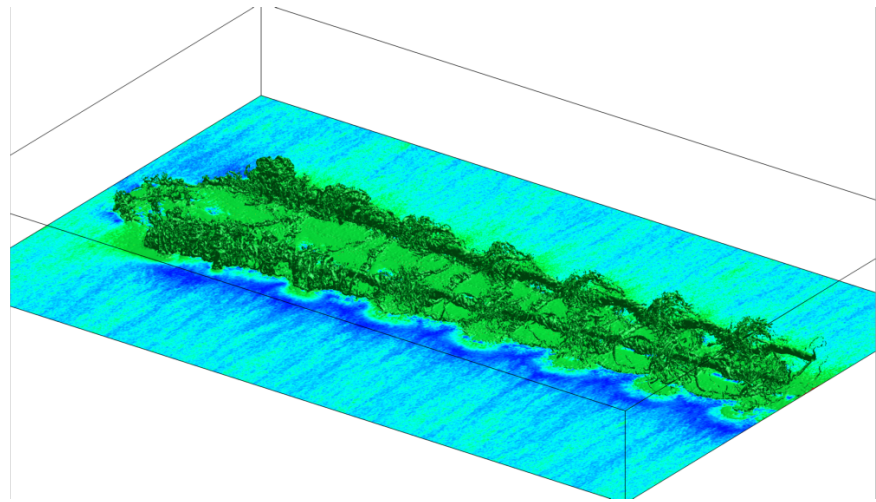

(b) $t^{*}=0.32$

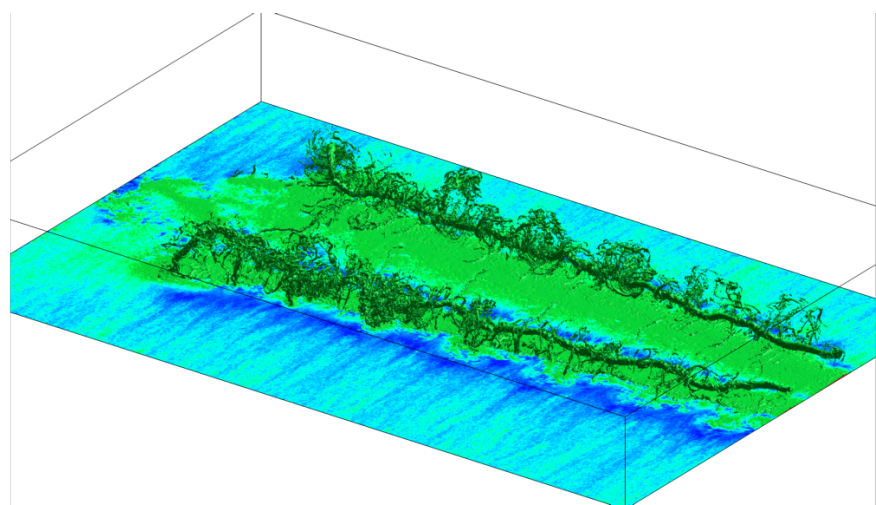

(d) $t^{*}=1.0$

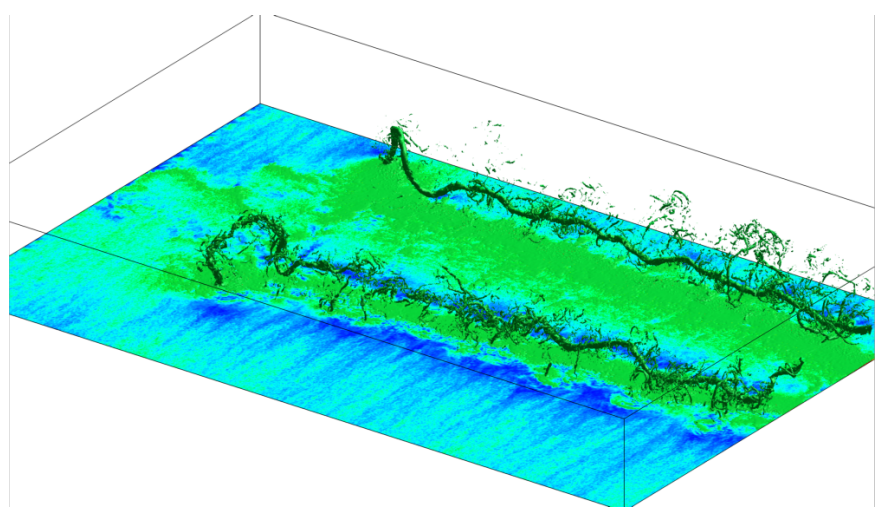

(f) $t^{*}=1.8$ 
Figure 12 Normalized vortex circulation development for the entire upwind vortex (left) and downwind vortex (right).

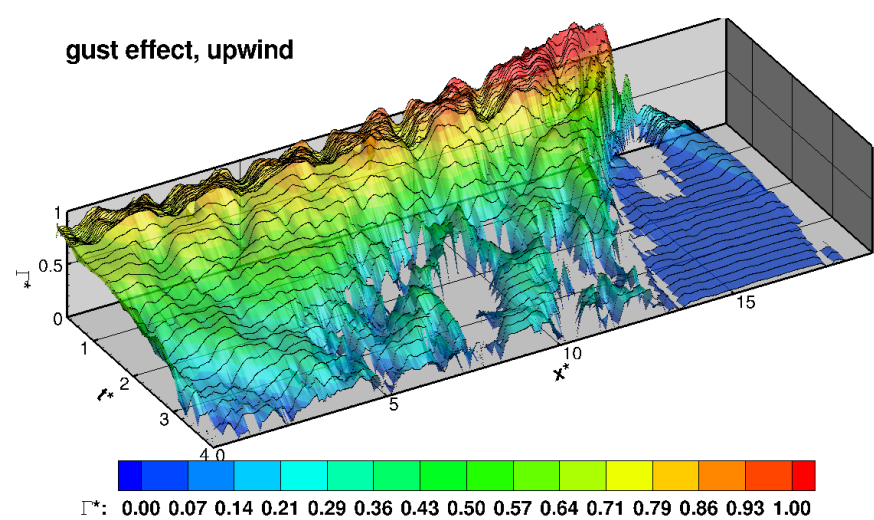

(a)

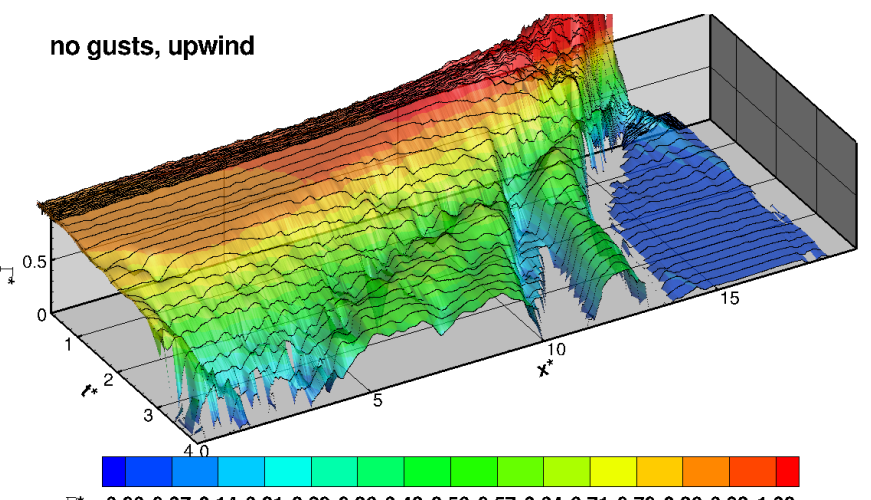

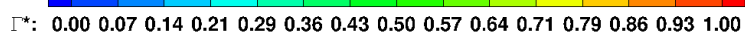

(c)

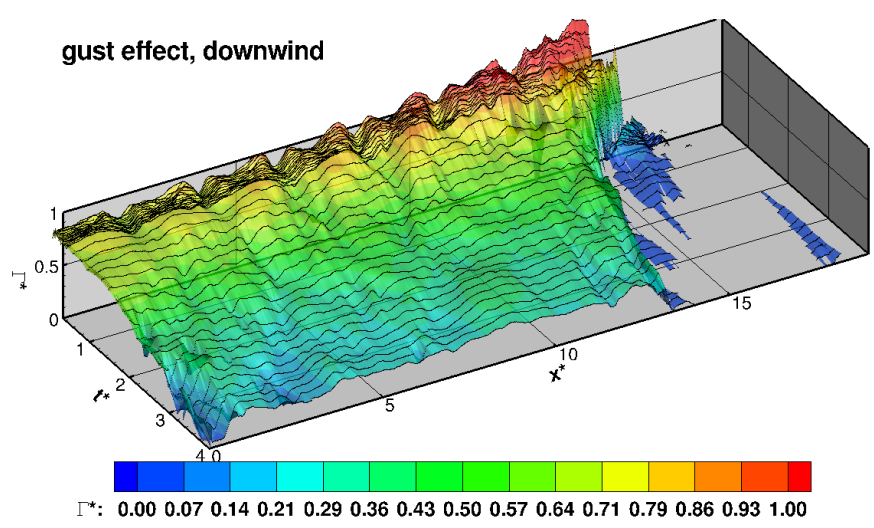

(b)

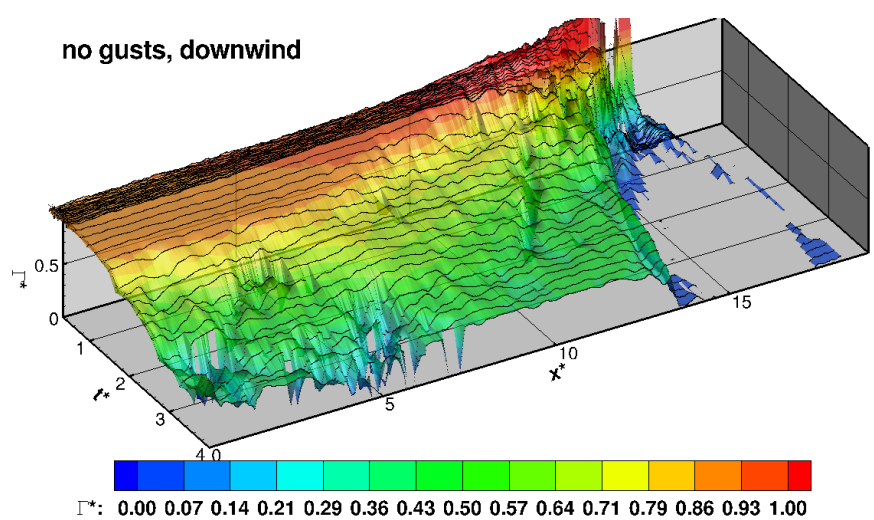

(d)

Figure 13 Wake vortex rebound height at different slices perpendicular to the flight direction.

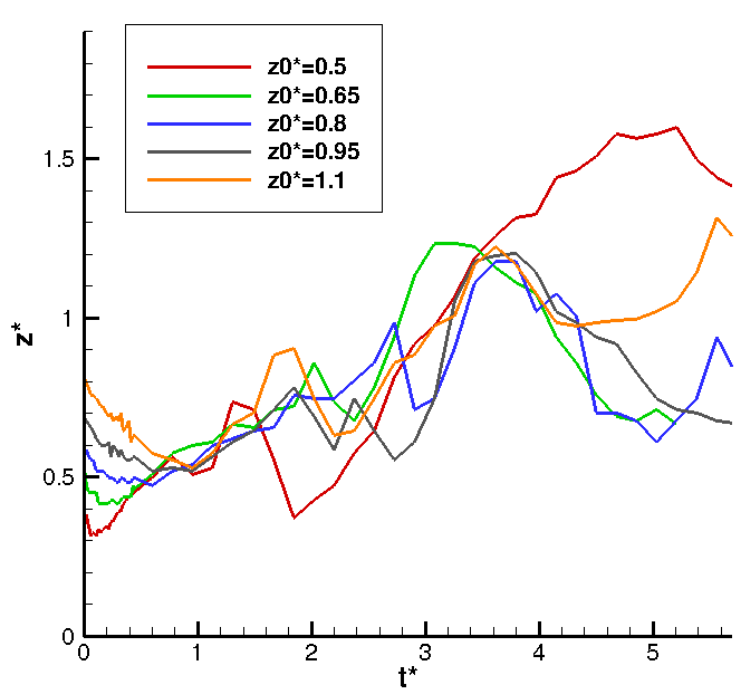

(a) upwind

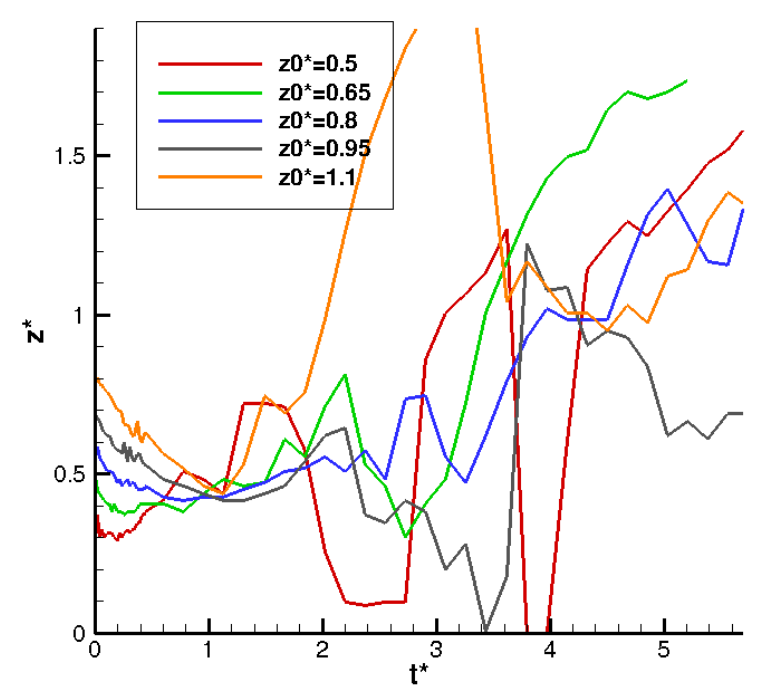

(b) downwind 